\title{
Quantitative assessment of the diagnostic role of mucin family members in pancreatic cancer: a meta-analysis
}

\author{
Shunda Wang, Lei You, Menghua Dai, Yupei Zhao \\ Department of General Surgery, Peking Union Medical College Hospital, Chinese Academy of Medical Sciences and Peking Union Medical \\ College, Beijing, China \\ Contributions: (I) Conception and design: Y Zhao, M Dai, S Wang; (II) Administrative support: L You; (III) Provision of study materials or patients: \\ None; (IV) Collection and assembly of data: S Wang, L You; (V) Data analysis and interpretation: S Wang, M Dai, Y Zhao; (VI) Manuscript writing: \\ All authors; (VII) Final approval of manuscript: All authors. \\ Correspondence to: Menghua Dai; Yupei Zhao, MD. Doctor, Professor, Department of General Surgery, Peking Union Medical College Hospital, No. 1, \\ Shuai Fu Yuan, Dongcheng District, Beijing 100730, China. Email: Daimh@pumch.cn; zhao8028@263.net.
}

Background: The use of mucins (MUC) as specific biomarkers for various malignancies has recently emerged. MUC1, MUC4, MUC5AC, and MUC16 can be detected at different stages of pancreatic cancer (PC), and can be valuable for indicating the initiation and progression of this disease. However, the diagnostic significance of the mucin family in patients with PC remains disputed. Herein, we assessed the diagnostic accuracy of mucins in PC using a meta-analysis.

Methods: We searched the PubMed, Cochrane Library, Institute for Scientific Information (ISI) Web of Science, Embase, and Chinese databases from their date of inception to June 1, 2020 to identify studies assessing the diagnostic performance of mucins in PC. The estimations of diagnostic indicators in selected studies were extracted for further analysis by Meta-DiSc software. Publication bias was assessed using Deeks' funnel plot asymmetry test.

Results: Our meta-analysis included 34 studies. The pooled accuracy indicators of MUC1 in PC including the sensitivity, specificity, diagnostic odds ratio (DOR), positive likelihood ratio (PLR), and negative likelihood ratio (NLR) (with 95\% confidence intervals) were 0.84 (0.82-0.86), 0.60 (0.56-0.64), 18.37 (9.18-36.78), 2.62 (1.79-3.86), and 0.22 (0.15-0.33), respectively. The area under the summary receiver operating characteristic (SROC) curve was 0.8875 and the $\mathrm{Q}$ index was 0.8181 . Quantitative randomeffects meta-analysis of MUC4 in PC using the summary (ROC) curve model revealed a pooled sensitivity of 0.86 (95\% confidence interval, $0.82-0.89$ ) and specificity of 0.88 (95\% confidence interval, 0.85-0.91). In addition, the meta-analysis of MUC5AC in PC diagnosis also showed a high sensitivity and specificity of 0.71 (95\% confidence interval, 0.65-0.76) and 0.60 (95\% confidence interval, 0.53-0.66), respectively. Regarding MUC16, the area under the summary ROC curve and Q index were 0.9185 and 0.8516 , respectively.

Conclusions: In summary, our results suggested a good diagnostic accuracy of several crucial mucins in PC. Mucins may serve as optional indicators in PC examination, and further research is warranted to investigate the role of mucins as potential clinical biomarkers.

Keywords: Pancreatic cancer (PC); MUC1; MUC4; MUC5AC; MUC16; diagnosis

Submitted Jul 31, 2020. Accepted for publication Nov 09, 2020.

doi: 10.21037/atm-20-5606

View this article at: http://dx.doi.org/10.21037/atm-20-5606

\section{Introduction}

Pancreatic cancer (PC) is the fourth leading cause of cancer-related death in the United States, with a dismal overall prognosis and a 5 -year survival rate of $8 \%$ (1). The only curative treatment for patients with $\mathrm{PC}$ is surgery; however the 5 -year survival rate of patients 
who undergo surgical resection is unfavorable (2). Due to the difficulty in diagnosing PC early, more than $80 \%$ of patients are diagnosed in the late stage, and only $20 \%$ of patients can undergo radical surgery with an initial diagnosis of resectable tumors (3). The clinical methods used to diagnose PC mainly rely on computed tomography, magnetic resonance imaging, endoscopic ultrasonography, and the detection of cancer biomarkers in peripheral blood. Commonly, serum carbohydrate antigen (CA)-199 is used to diagnose PC (4); however, the area under the curve (AUC) is 0.7 when CA-199 is used to discriminate patients with PC from healthy controls (5). Serum CA125, alpha fetoprotein (AFP), and carcinoembryonic antigen (CEA) are also important indices for PC assessment, but their sensitivities are inferior to that of CA19-9 $(6,7)$. Consequently, better PC treatment requires identifying novel diagnostic markers to facilitate early detection and improve prognosis in patients with PC.

Mucins (or MUC) are a family of high-molecular-weight glycosylated proteins, which form a protective barrier of epithelial cells (8). At present, more than 20 mucins have been identified. Mucins can be divided into two groups according to their structure and function: transmembrane mucins (MUC1, MUC4, MUC12, and MUC16) and secreting mucins (MUC2, MUC5AC, and MUC6) $(9,10)$. The subcellular distribution and expression level of mucins change according to the different disease stages, indicating their roles in tumorigenesis, malignant transformation, and cancer progression (11). The expression level of mucins has been shown to participate in the progression and metastasis of different cancers, including colon carcinoma, ovarian malignancy, renal tumors, breast cancer, and lung carcinoma (9,12-15).

With regards to $\mathrm{PC}$, mucins have been demonstrated to be strongly involved in carcinogenesis and progression, suggesting that the abnormal expression of mucins might be a predictive marker in PC (16). The correlation between MUC1 and PC has been explored in many studies. MUC1 is a major component of ductal cells in healthy pancreatic tissue but is aberrantly expressed in PC cells. MUC1 exposes tumor-associated epitopes and provokes cellular and humoral immune responses. MUC1 is expressed in more than $60 \%$ of $\mathrm{PC}$ cases, and its high expression is correlated with poor prognosis (17). Moreover, studies have demonstrated that MUC4 is involved in several oncogenic properties and has been shown to be expressed in $32 \%$, $89 \%$, and $79 \%$ of PC cases in different studies, respectively
(18-20). Furthermore, when combined with CA19-9, MUC5AC has been reported to improve the diagnostic sensitivity of pancreatic malignancies (21). Moreover, the expression level of MUC16 increases from low-grade to high-grade dysplasia in pancreatic tissue (22).

However, findings regarding the application of mucins as a diagnostic indicator of PC remain conflicting. Systematic analyses of these data might be valuable to verify the diagnostic accuracy of mucin family members in PC. Therefore, the purpose of our quantitative metaanalysis was to investigate the diagnostic potential of mucin family members that have been widely explored in PC (MUC1, MUC4, MUC5AC, and MUC16), which has not been reported previously. We present the following article in accordance with the preferred reporting items for systematic reviews and meta-analyses (PRISMA) reporting checklist (23) (available at http://dx.doi.org/10.21037/atm20-5606).

\section{Methods}

\section{Search strategy}

We performed systematic literature searches of the PubMed, Institute for Scientific Information (ISI) Web of science, the Cochrane Library, and Embase databases, along with the Wanfang and China National Knowledge Infrastructure databases, from the date of initiation of the database to June 2020. The primary aim of our literature search was to access original articles that focused on mucins in the diagnosis of PC. Keywords included ("mucin" OR "mucins" OR "MUC") AND ("pancreatic cancer" OR "pancreatic carcinoma" OR "pancreatic tumor" OR "pancreatic neoplasm") AND ("diagnostic "OR "diagnosis"). References cited by the selected literatures were also manually searched to find additional studies.

\section{Inclusion and exclusion criteria}

The inclusion criteria for studies were as follows: (I) research about the detection of PC with MUC by immunohistochemistry; (II) cases pathologically confirmed as PC (the gold standard criterion); (III) complete quadruplex table data available from the full text or abstract of the literature to calculate the diagnostic parameters; (IV) inclusion of a control group with healthy or benign patients. Studies were excluded based on the following 
criteria: (I) those reporting on malignancies other than PC or metastatic PC; (II) cases confirmed with nonpathological evidence; (III) MUC detected by methods other than immunohistochemistry; (IV) lack of diagnostic quadruple table data; (V) case reports or review articles. All included publications were independently evaluated by two reviewers, and discrepancies were resolved through discussion and consensus.

\section{Data extraction and quality assessment}

Two reviewers independently examined eligible studies and extracted data concerning the author, country, year of publication, case numbers, MUC phenotype, detection method, and histological type with the true-positive (TP), true-negative (TN), false-positive (FP), and false-negative (FN) results. Each study was assessed by two independent researchers for inclusion or exclusion, and discrepancies were resolved by a third investigator for re-evaluation. The quality of included articles was scored by the 14 items Quality Assessment of Diagnostic Accuracy Studies (QUADAS) tool (24).

\section{Diagnostic efficiency index}

The diagnostic efficiency evaluation indexes used were sensitivity, specificity, diagnostic odds ratio (DOR), positive likelihood ratio (PLR), negative likelihood ratio (NLR), area under the curve (AUC) of the summary receiver operating characteristic (SROC) curve, and the Q index. An AUC close to 1 implies a good differential ability to separate patients from healthy controls. Also, a higher Q index indicates a higher accuracy of the diagnostic test.

\section{Statistical analysis}

The standard protocol recommended for the metaanalysis of diagnostic accuracy was adopted (25). Data such as diagnostic quadruple tables and critical values were extracted and entered into the Meta-DiSc 1.4 software (26). Heterogeneity caused by the threshold effect was checked by Spearman's correlation analysis. $\mathrm{I}^{2}>50 \%$ indicated the presence of heterogeneity in the studies, and a random effects model was applied in the pooled analysis. A fixed effects model was used to aggregate the accuracy indicators $(27,28)$, while $95 \%$ confidence intervals (CIs) were used for all pooled data. $\mathrm{P}$ values are two-tailed, and a $\mathrm{P}$ value $<0.05$ was considered statistically different. Deeks' funnel plot was employed to assess publication bias. Meta-DiSc 1.4 and Stata 12.0 were employed to perform the analyses.

\section{Results}

\section{Search results}

After a comprehensive literature search to identify related studies published before June 2020, 746 records were initially screened for inclusion. A further 17 studies were acquired through other sources. After excluding 194 duplicates, we screened the abstracts of 569 studies, and excluded 434 that did not meet the inclusion criteria. We also excluded articles that were unrelated to our main subject, along with abstracts, reviews, case reports, and nonEnglish or non-Chinese language articles. The full texts of the remaining 135 studies were further evaluated. Of these, 101 articles were excluded due to lack of sufficient data to calculate sensitivity and specificity, overlapped data, or lack of control groups in the study. Finally, 34 observational studies involving 3,900 patients were included in our review $(18,19,29-40)$. The study selection process is detailed in Figure 1 (41-60).

\section{Study characteristics}

The basic information of all included studies is summarized in Tables 1,2. In total, 34 eligible studies published between 1993 and 2019 were included. No prospective or randomized controlled trials (RCTs) were included. All included studies evaluated the expression level of MUC in both PC and negative controls using immunohistochemistry. There were 23 studies that investigated the diagnostic accuracy of MUC1 for PC, 8 studies that reported on MUC4, 9 studies that reported on MUC5AC, and 4 studies that reported on MUC16. The QUADAS tool was employed to assess the quality of the studies, and the final score of each study is shown in Tables 1 and 2.

\section{Quantitative data analysis of MUC1}

Twenty-three studies involving 1,797 patients provided data for this analysis. Spearman's correlation coefficient results yielded an rs of 0.404 and a $\mathrm{P}$ of 0.069 , suggesting that sensitivity and specificity were positively correlated, and therefore, there was no threshold effect. Also, the heterogeneity test results suggested that there was heterogeneity in the sensitivity $\left(\mathrm{I}^{2}=86.9 \%\right)$, specificity 


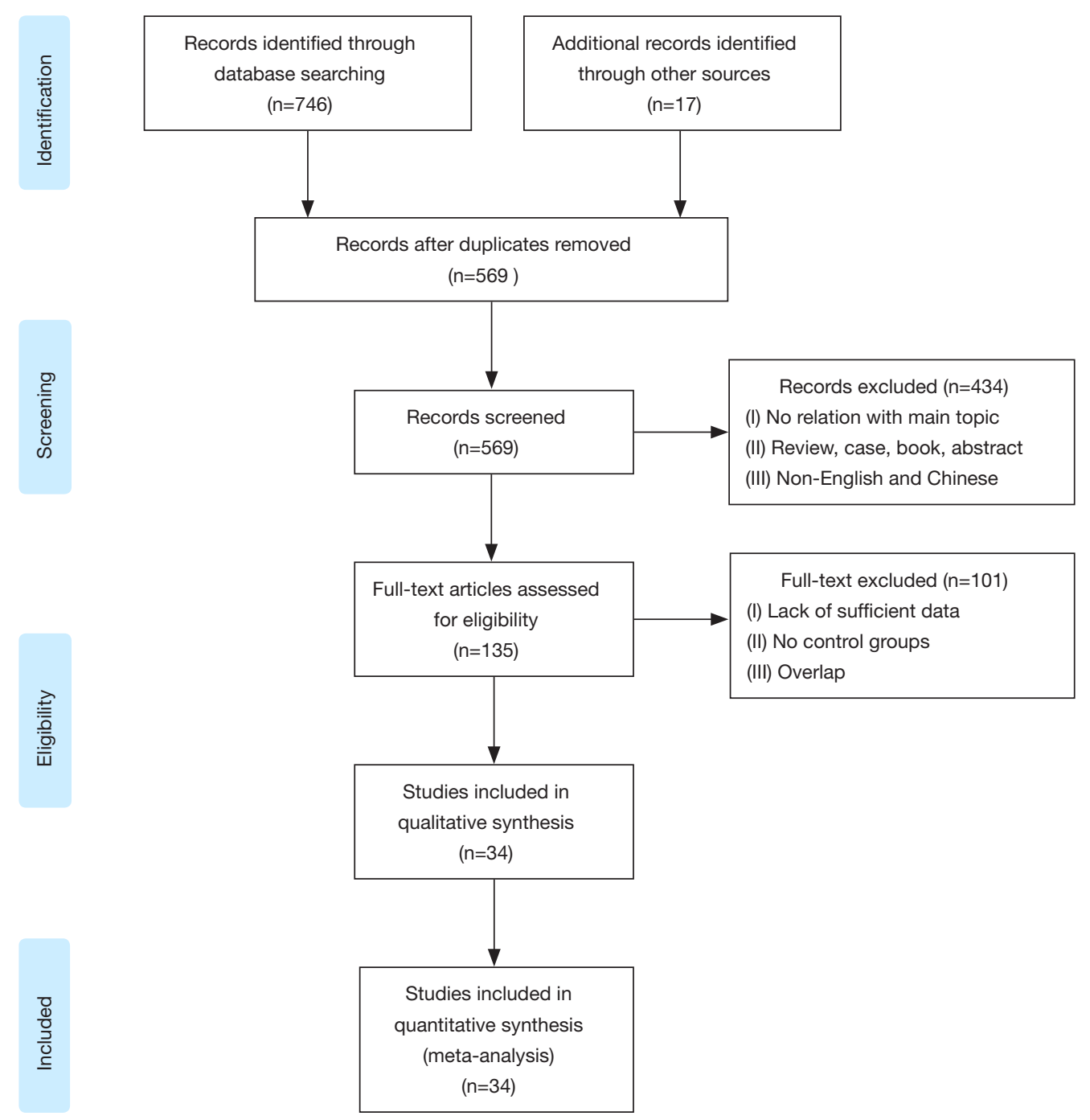

Figure 1 Flow diagram of included studies.

$\left(\mathrm{I}^{2}=91.6 \%\right)$, and DOR $\left(\mathrm{I}^{2}=72.4 \%\right)$ among the studies, so the random effects model was employed to combine effect quantities. The pooled sensitivity and specificity of MUC1 in the diagnosis of PC were 0.84 (95\% CI, 0.82-0.86) and 0.60 (95\% CI, 0.56-0.64), respectively. The PLR, NLR, and DOR were 2.62 (95\% CI, 1.79-3.86), 0.22 (95\% CI, $0.15-0.33$ ), and 18.37 (95\% CI, 9.18-36.78), respectively. The recalculated sensitivity and specificity are shown in Figure 2, and the PLR, NLR, and DOR for MUC1 in PC diagnosis are shown in Figure 3. The AUC was 0.8875 and the $\mathrm{Q}$ index was 0.8181 . The SROC graph displays the summary values of sensitivity and specificity of MUC1 in
PC diagnosis (Figure 4).

\section{Quantitative data analysis of MUC4}

Eight studies containing 909 cases investigated the diagnostic accuracy of MUC4 in PC. A random effects model was used, and the pooled sensitivity and specificity were 0.86 (95\% CI, 0.82-0.89) and 0.88 (95\% CI, 0.85$0.91)$, respectively. In addition, the PLR, NLR, and DOR were 6.95 (95\% CI, 2.33-20.69), 0.20 (95\% CI, 0.14-0.30), and 36.64 (95\% CI, 9.49-141.46), respectively. The pooled DOR was 36.64 (95\% CI, 9.49-141.46). The Q index was 
Table 1 Characteristics of included studies of MUC1 for the detection of pancreatic cancer

\begin{tabular}{|c|c|c|c|c|c|c|c|c|c|c|}
\hline Author & Year & Country & Method & Blind & Patient number & $\mathrm{TP}(\mathrm{a})$ & $\mathrm{FP}(\mathrm{b})$ & $\mathrm{FN}(\mathrm{c})$ & $\mathrm{TN}(\mathrm{d})$ & Quality score \\
\hline Terada & 1996 & Japan & $\mathrm{IHC}$ & No & 52 & 25 & 25 & 0 & 2 & 10 \\
\hline Masak & 1999 & Japan & $\mathrm{IHC}$ & No & 64 & 55 & 3 & 0 & 6 & 9 \\
\hline Luttges & 2001 & Germany & $\mathrm{IHC}$ & No & 84 & 35 & 20 & 0 & 29 & 10 \\
\hline Kim & 2002 & USA & $\mathrm{IHC}$ & No & 195 & 51 & 94 & 13 & 37 & 10 \\
\hline Terris & 2002 & France & $\mathrm{IHC}$ & No & 147 & 79 & 11 & 11 & 46 & 12 \\
\hline Yonezawa & 2002 & Japan & $\mathrm{IHC}$ & No & 79 & 44 & 4 & 2 & 29 & 9 \\
\hline Chhieng & 2003 & USA & $\mathrm{IHC}$ & No & 35 & 23 & 1 & 1 & 10 & 11 \\
\hline Ueda & 2005 & Japan & $\mathrm{IHC}$ & No & 45 & 21 & 6 & 0 & 18 & 11 \\
\hline Zhang & 2005 & China & $\mathrm{IHC}$ & No & 82 & 40 & 10 & 12 & 20 & 9 \\
\hline Gao & 2006 & China & $\mathrm{IHC}$ & No & 58 & 29 & 3 & 14 & 12 & 9 \\
\hline Giorgadze & 2006 & USA & $\mathrm{IHC}$ & No & 43 & 25 & 7 & 5 & 6 & 8 \\
\hline Ohuchida & 2006 & Japan & $\mathrm{IHC}$ & No & 62 & 23 & 18 & 0 & 21 & 12 \\
\hline Okada & 2006 & Japan & $\mathrm{IHC}$ & No & 47 & 9 & 3 & 5 & 30 & 11 \\
\hline Wang & 2007 & China & $\mathrm{IHC}$ & No & 54 & 31 & 4 & 7 & 12 & 10 \\
\hline Sabrina & 2013 & USA & $\mathrm{IHC}$ & No & 57 & 30 & 26 & 0 & 1 & 9 \\
\hline
\end{tabular}

IHC, immunohistochemistry; TP, true positive; FP, false positive; FN, false negative; TN, true negative.

0.8353 and the AUC was 0.9038 (Figure 5).

\section{Quantitative data analysis of MUC5AC}

Nine articles including 515 cases were analyzed for the accuracy of MUC5AC expression for PC diagnosis. Pooled analyses revealed heterogeneity in the included research, so a random effects model was utilized. Results showed that the sensitivity and specificity of MUC5AC were 0.71 (95\% CI, 0.65-0.76) and 0.60 (95\% CI, 0.53-0.66), respectively. Moreover, the PLR, NLR, and DOR were 1.81 (95\% CI, 0.81-4.11), 0.41 (95\% CI, 0.16-1.06), and 6.18 (95\% CI, 0.81-47.36), respectively. The SROC curve showed that the
AUC was 0.7735 and the Q index was 0.7131 (Figure 6).

\section{Quantitative data analysis of MUC16}

MUC16 was mentioned in four articles. Due to heterogeneity, a random effects model was employed to analyze the combined effect quantities. The final pooled values of sensitivity, specificity, and DOR were 0.72 (95\% CI, 0.67-0.76), 0.87 (95\% CI, 0.83-0.90), and 32.94 (95\% CI, 1.51-717.78), respectively. The PLR and NLR were 12.16 (95\% CI, 0.56-266.28) and 0.36 (95\% CI, 0.17-0.78), respectively. The area under the SROC curve was 0.9185 and the $\mathrm{Q}$ index was 0.8516 (Figure 7). 
Table 2 Main characteristics of the included publications about MUC4, MUC5AC, and MUC16

\begin{tabular}{|c|c|c|c|c|c|c|c|c|c|c|}
\hline Author & Year & Country & Method & Blind & Patient number & $\mathrm{TP}(\mathrm{a})$ & $\mathrm{FP}(\mathrm{b})$ & $\mathrm{FN}(\mathrm{c})$ & $\mathrm{TN}(\mathrm{d})$ & Quality score \\
\hline \multicolumn{11}{|l|}{ MUC4 } \\
\hline Mahefatiana & 2001 & USA & $\mathrm{IHC}$ & No & 26 & 12 & 0 & 4 & 10 & 10 \\
\hline Michael & 2002 & USA & $\mathrm{IHC}$ & No & 274 & 25 & 0 & 3 & 246 & 12 \\
\hline Park & 2003 & USA & $\mathrm{IHC}$ & No & 171 & 65 & 8 & 17 & 81 & 11 \\
\hline Atul & 2007 & USA & $\mathrm{IHC}$ & No & 89 & 55 & 4 & 16 & 14 & 9 \\
\hline Sabrina & 2013 & USA & $\mathrm{IHC}$ & No & 55 & 24 & 17 & 5 & 9 & 9 \\
\hline Marek & 2016 & Poland & $\mathrm{IHC}$ & No & 141 & 91 & 26 & 10 & 14 & 10 \\
\hline Carlos & 2017 & Sweden & $\mathrm{IHC}$ & No & 88 & 73 & 0 & 5 & 10 & 11 \\
\hline Yonezawa & 2002 & Japan & $\mathrm{IHC}$ & No & 56 & 32 & 7 & 8 & 9 & 9 \\
\hline Hiroyuki & 2004 & Japan & $\mathrm{IHC}$ & No & 62 & 5 & 20 & 33 & 4 & 9 \\
\hline Ohuchida & 2006 & Japan & $\mathrm{IHC}$ & No & 43 & 29 & 8 & 1 & 5 & 12 \\
\hline Giorgadze & 2006 & USA & $\mathrm{IHC}$ & No & 71 & 15 & 30 & 23 & 3 & 8 \\
\hline Wang & 2007 & China & $\mathrm{IHC}$ & No & 56 & 32 & 7 & 8 & 9 & 10 \\
\hline Sabrina & 2013 & USA & $\mathrm{IHC}$ & No & 88 & 34 & 0 & 12 & 42 & 9 \\
\hline Marek & 2016 & Poland & $\mathrm{IHC}$ & No & 57 & 25 & 5 & 5 & 22 & 10 \\
\hline Meritxell & 2018 & Portugal & $\mathrm{IHC}$ & No & 24 & 19 & 0 & 2 & 3 & 9 \\
\hline
\end{tabular}

IHC, immunohistochemistry; TP, true positive; FP, false positive; FN, false negative; TN, true negative.

\section{Publication bias}

Deeks' funnel plot asymmetry test was carried out to assess the publication bias. The result indicated that there was no obvious publication bias present $(\mathrm{P}=0.47)$ (Figure 8).

\section{Discussion}

PC manifests with extremely malignant effects and a high mortality rate. According to the latest epidemiological data from the United States, the 5-year survival rate of PC is less than $10 \%$ (2). Patients with PC have a dismal prognosis, partially due to delays in diagnosis. The most widely applied clinical detection method is immunological analysis of serum tumor markers, such as CA19-9, CEA, and AFP glycoproteins. These belong to tumor-associated antigens, which are widely expressed in the digestive, urinary, and respiratory tracts of PC patients, as well as in pancreatitis and benign pancreatic lesions, and thus lack specificity (61). Consequently, it is necessary to identify novel diagnostic markers to facilitate a breakthrough in the accurate diagnosis of $\mathrm{PC}$, which will lead to improvements in the poor outcomes of PC patients. To the best of our knowledge, this is the first meta-analysis to focus on mucin family expression for the diagnosis of PC based on 
A



B

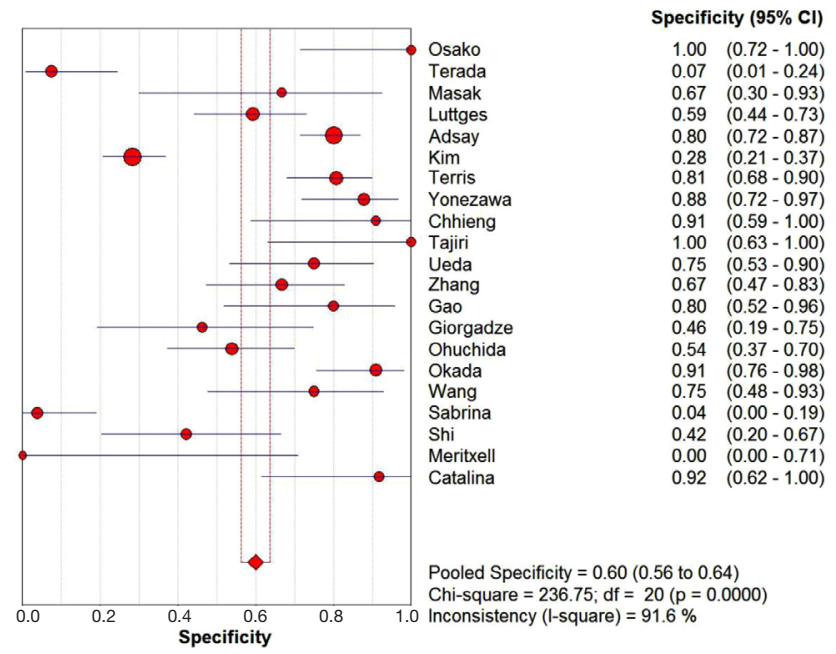

Figure 2 Forest plot of pooled sensitivity (A) and specificity (B) of MUC1 for the diagnosis of PC. PC, pancreatic cancer.

A



B

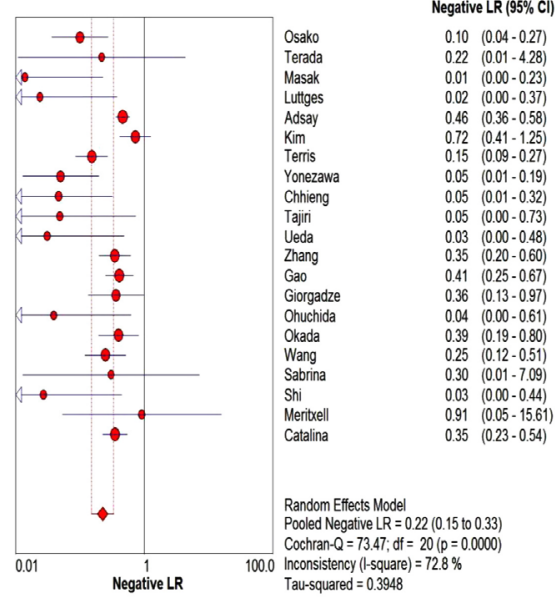

C

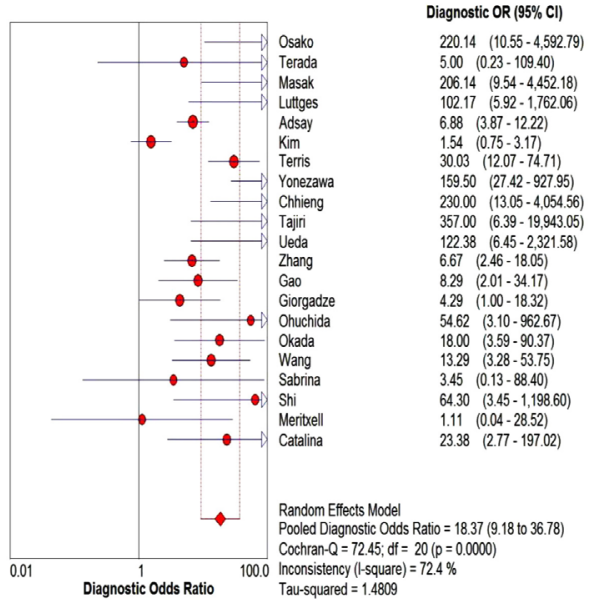

Figure 3 Forest plot of pooled PLR (A), NLR (B), and DOR (C) of MUC1 for the diagnosis of PC. PC, pancreatic cancer; DOR, diagnostic odds ratio; PLR, positive likelihood ratio; NLR, negative likelihood ratio.

published reports.

MUC1, a heterodimeric oncoprotein, is aberrantly upregulated in various malignancies, including PC $(14,62,63)$. Previous studies have revealed that MUC1 is highly expressed in PC tissues and exerts an important role in the oncogenesis of PC, participating in several signaling pathways such as hypoxia-inducible factor-1, Slug, AKT, mitogen-activated protein kinase, and $\mathrm{Wnt} / \beta$-catenin $(10,64)$. It has already been confirmed that the level of MUC1 expression is elevated in PC tissues compared to negative controls (30). In addition, MUC1 is involved in epithelial-mesenchymal transition, the process through which tumor cells acquire their invasive potential $(65,66)$. In pathological cases, such as those of colon cancer and stomach carcinoma, MUC1expression has been found vary in expression (63). Our meta-analysis is the most comprehensive study that supports MUC1 as a potential diagnostic marker based on quantitative assessments in patients with PC. MUC1 could be used to differentiate patients with PC from controls and manifested a pooled 
sensitivity of 0.84 (95\% CI, $0.82-0.86$ ), a specificity of 0.60 (95\% CI, 0.56-0.64), an AUC of 0.8875, and a Q index of 0.8181 , demonstrating its potential diagnostic value. The AUC is regarded as the overall test performance, and an AUC of the SROC curve of approximately 1 suggests excellent diagnostic accuracy (67). Taken together, although the specificity of MUC1 was moderate, the sensitivity was high, and the Q index ranging from 0.7 to 0.9 indicated a

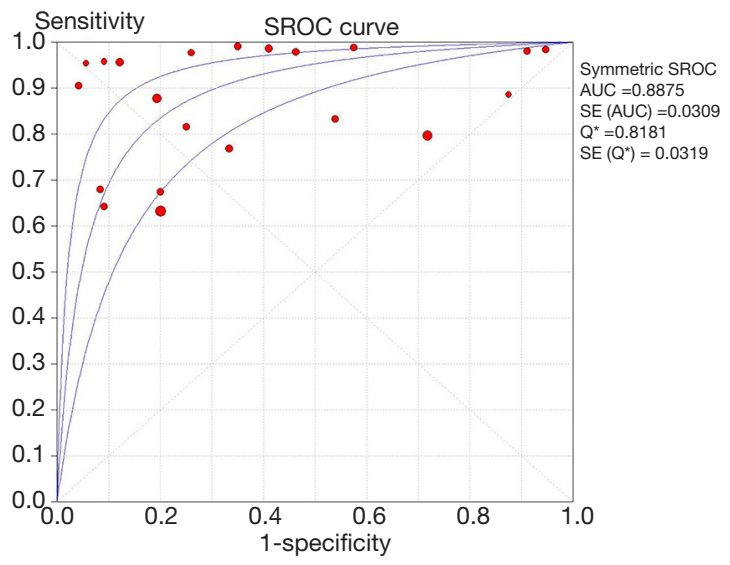

Figure 4 Summary receiver operating characteristic curve of MUC1 for the diagnosis of PC. PC, pancreatic cancer. good accuracy for diagnosing PC.

Regarding MUC4, de novo expression has been observed in PC but not in healthy tissue (16,52). MUC4 participates in the oncogenesis process by enhancing cellular growth, differentiation, and immune recognition, primarily via its transmembrane ligand for the receptor tyrosine kinase, ErbB2 $(68,69)$. In the present study, MUC4 had a high PLR of 6.95 (95\% CI, 2.33-20.69) and a low NLR of 0.20 (95\% CI, 0.14-0.30), which implies that it performed well in excluding cancer within the pancreas. In addition, MUC4 expression was also verified as an indicator for overall survival in patients with PC receiving gemcitabinebased chemotherapy and as a novel tumor antigen in PC immunotherapy $(54,70)$. In our analysis, MUC4 performed better than MUC5AC, with a higher sensitivity and Q index.

MUC5AC was first identified as an overexpressed gene in PC tissue compared with benign controls by IacobuzioDonahue et al. in 2003 (71). Moreover, the messenger ribonucleic acid level of MUC5AC in pancreatic juice exhibited good diagnostic performance in identifying PC (38). A panel of MUC3, MUC5AC, and MUC6 provided the ability to discriminate $\mathrm{PC}$ from a normal pancreas (50). Clinically, Kaur et al. investigated the
A

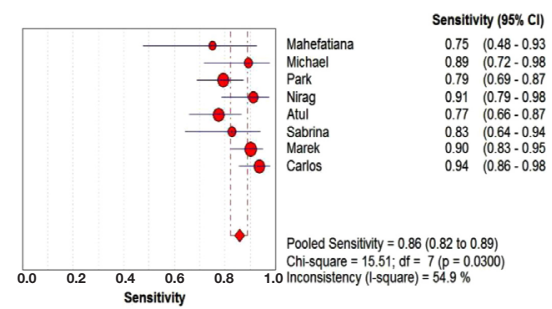

D

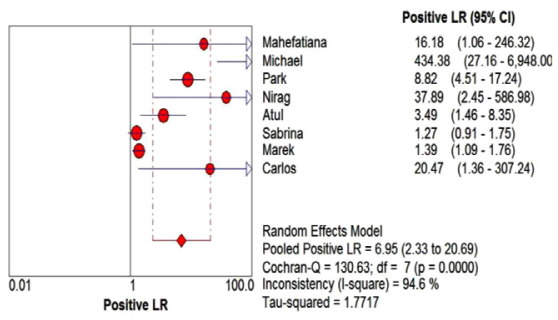

B

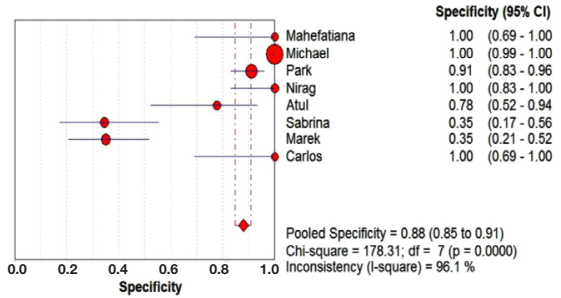

$\mathrm{E}$

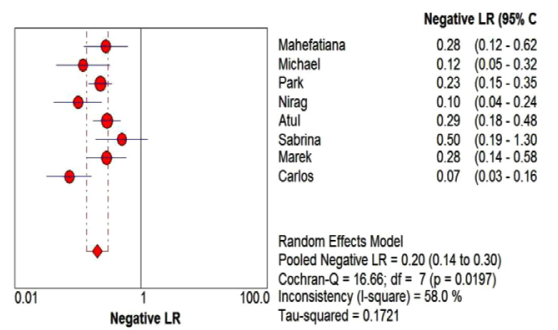

C
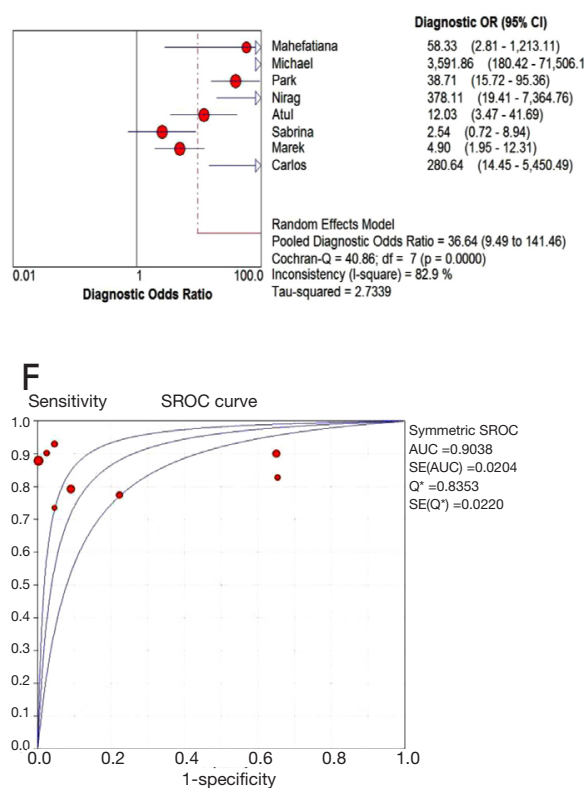

Figure 5 Forest plot of pooled sensitivity (A), specificity (B), DOR (C), PLR (D), NLR (E), and SROC (F) of MUC4 for the diagnosis of PC. PC, pancreatic cancer; DOR, diagnostic odds ratio; PLR, positive likelihood ratio; NLR, negative likelihood ratio; SROC, summary receiver operating characteristic. 
A

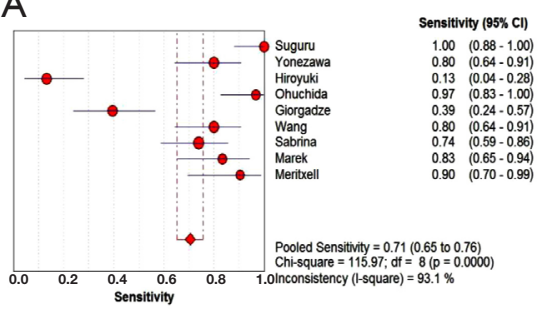

D

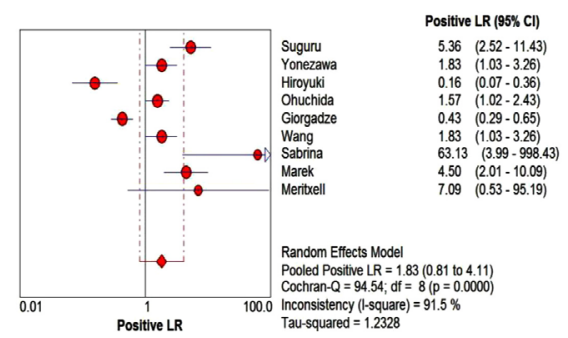

B

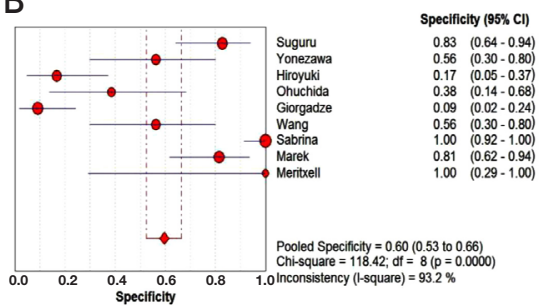

$E$



C

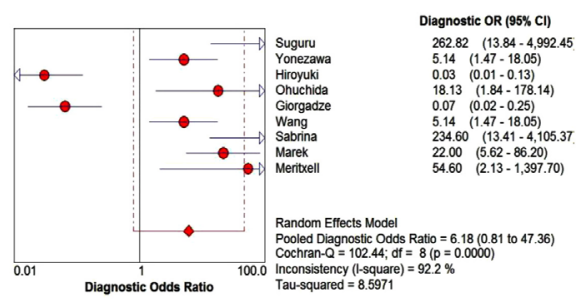

$\mathrm{F}$

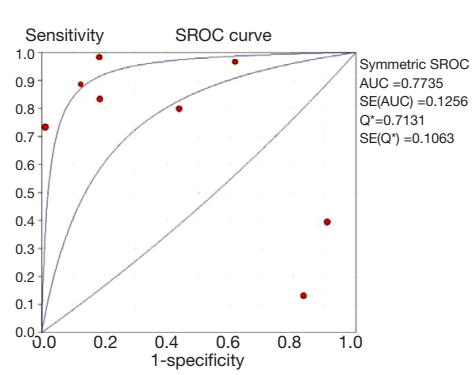

Figure 6 Forest plot of pooled sensitivity (A), specificity (B), DOR (C), PLR (D), NLR (E), and SROC (F) of MUC5AC for the diagnosis of PC. PC, pancreatic cancer; DOR, diagnostic odds ratio; PLR, positive likelihood ratio; NLR, negative likelihood ratio; SROC, summary receiver operating characteristic.

A

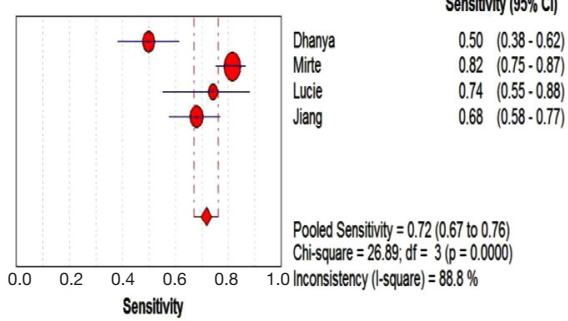

D

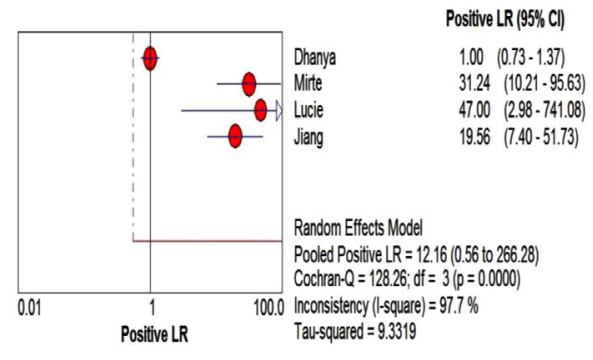

B

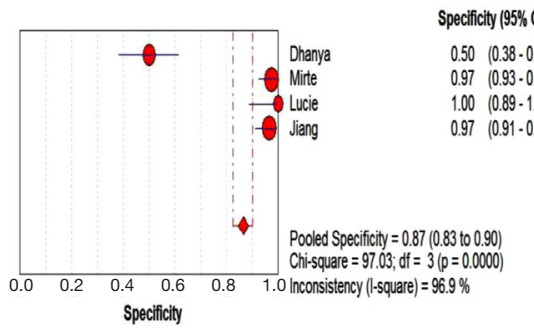

$\mathrm{E}$

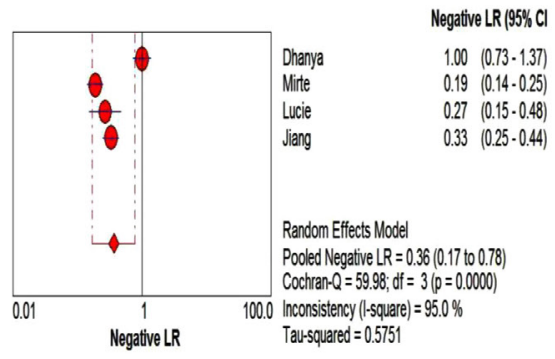

C

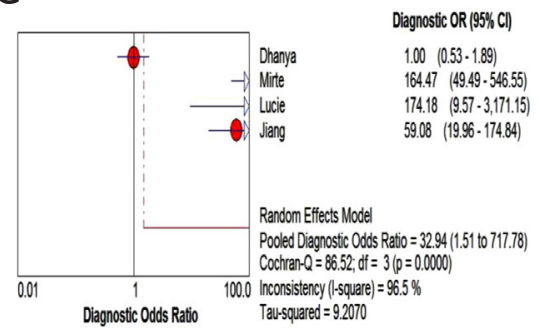

$\mathrm{F}$

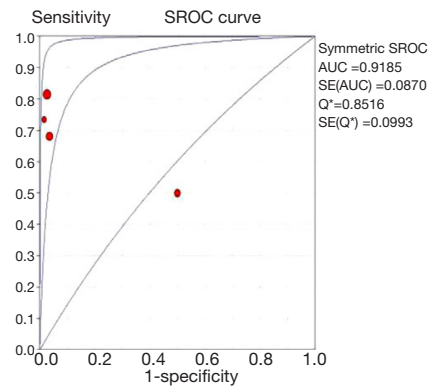

Figure 7 Forest plot of pooled sensitivity (A), specificity (B), DOR (C), PLR (D), NLR (E), and SROC (F) of MUC16 for the diagnosis of PC. PC, pancreatic cancer; DOR, diagnostic odds ratio; PLR, positive likelihood ratio; NLR, negative likelihood ratio; SROC, summary receiver operating characteristic. 


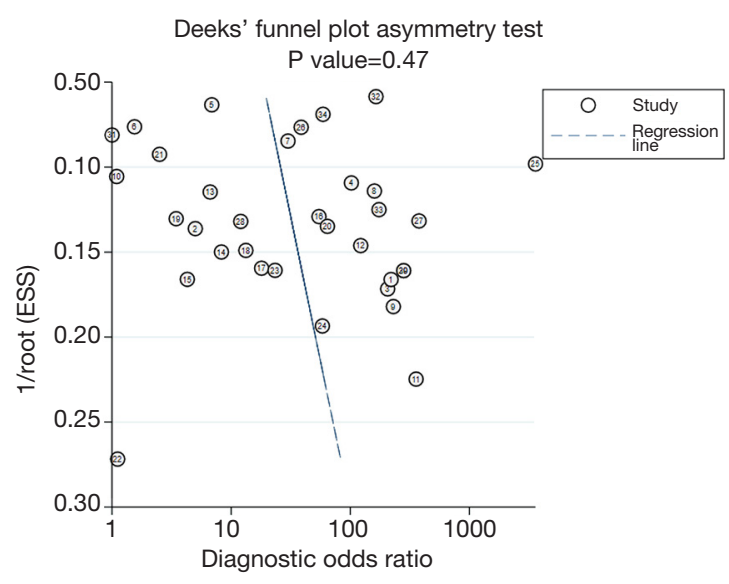

Figure 8 Detection of publication bias.

potential of MUC5AC in combination with CA199 to improve the diagnostic accuracy in a Caucasian population (21). In addition, Zhang et al. showed that the same panel could also benefit the accurate diagnosis of PC in Asian individuals (72). In addition to early diagnosis, MUC5AC also presented the ability to monitor the response to chemo/radiotherapy in patients with $\mathrm{PC}$.

Moreover, molecular cloning of CA125 led to the discovery of MUC16 in 2001 (73). MUC16 is used as a specific marker not only for ovarian malignancy, but also in gastric carcinoma, colorectal cancer, cholangiocarcinoma, and more recently in PC $(57,74)$. The diagnostic dilemma of MUC16 application as a diagnostic biomarker for PC is based on its presence in patients with benign pancreatic neoplasms and pancreatitis (58). Our results showed a sensitivity of 0.72 (95\% CI, $0.67-0.76)$ and specificity of 0.87 (95\% CI, 0.83-0.90) for MUC16. The AUC of the SROC was 0.9185 , which was approximately 1 , indicating its diagnostic potential. However, the number of included studies might not have been adequate.

Mucins not only serve as diagnostic markers, but also participate in important biological processes, including adhesion, immune regulation, chemoresistance, and intracellular signaling transductions $(10,54,75)$. As for CA19-9, which is the most popular tumor biomarker in the diagnosis of PC, its epitope is produced only on the MUC$1 / \mathrm{Y}$ core protein, indicating that the CA19-9 epitope may be a specific marker for the MUC-1/Y protein (76). The expression of mucins is accompanied by the evolution of the disease, with its level changing in accordance with the progression of healthy tissue to precursor lesions to PC. Moreover, mucins can also regulate signal pathways such as p53, PI3K-AKT (phosphatidylinositide-3-kinase/AKT), JAK-STAT (Janus kinase-signal transducer and activators of transcription), RAS-ERK (Ras-extracellular signalregulated kinase), and MAP (mitogen-activated protein) kinase (75). In some studies, mucins have been reported to be associated with clinicopathological characteristics such as histological pattern, tumor location, or vascular invasion $(77,78)$. Moreover, some mucins have also been significantly correlated with the prognosis of PC patients $(79,80)$.

The combination of mucin panels provides considerable functional value in differentiated diagnosis. For instance, MUC1 to MUC6 expression in lung carcinoma was correlated with tumor differentiation and histologic subtypes, while MUC5AC was found capable of differentiating primary lung cancer from metastatic PC in the lung (81). As for colon cancer, several studies detected the MUC1, MUC2, MUC5AC, and MUC6 expression using tissue microarrays and demonstrated their clinical significance $(82,83)$. In PC, Dai et al. (84) demonstrated that MUC14 plus MUC15 serves as a prognostic marker for stomach adenocarcinoma through bioinformatics analysis. As can be seen, mucin combinations have frequently been applied in clinical diagnosis. However, there is currently no commercial mucin chip. A more in-depth investigation of mucin panels may produce the means to provide an early diagnosis of different cancers.

Some limitations to our study should also be noted. (I) The studies included in this meta-analysis were observational or retrospective studies, while the RCTs regarding pathological staining lack real-world value. Furthermore, there were only a few studies related to this topic, and the data presented in these articles did not correspond to large sample sizes, making them prone to produce erroneous conclusions. Future larger sample size studies and results are needed to verify this conclusion. (II) There was inevitably a selection bias in the published literature. In all included studies, the interpretation of mucin expression was dependent on the gold standard; however, there was a lack of blinding. (III) Although there was no threshold effect, the threshold settings of the included studies were different. The results of the immunohistochemistry tests were evaluated by combining the percentage of colored cells and the grading of coloring intensity. However, in some studies, the results were evaluated only based on the numerical value of the percentage of colored cells. The standards and methods of each study were different and could not be unified; therefore, we adopted a random effects model 
in circumstances where heterogeneity existed between the studies. We also conducted the Deeks' funnel plot asymmetry test to assess the publication bias.

Overall, the detection of mucin family expression offers potential value for the diagnosis of PC. Clinically, the expression level of mucins in PC tissue may also function as a prognostic marker and therapeutic target in the future. Further analyses must be conducted to calculate mucin levels with clinicopathological parameters to comprehensively ascertain the role of mucins in PC.

\section{Conclusions}

Our meta-analysis is the first study to examine the value of mucins in the diagnosis of PC. We demonstrated that mucins yield acceptable sensitivity and specificity for distinguishing patients with PC from healthy controls, which indicates that they may be a potential innovative biomarker for PC diagnosis. Considering the limitations in our analysis, further scientific studies with larger sample sizes and high-quality evidence are needed to evaluate this topic more accurately.

\section{Acknowledgments}

Funding: This study's design, collection, analysis, writing, and interpretation were supported by the Application and Promotion of Capital Special Clinical Research Project of the Beijing Municipal Science \& Technology Commission (No. Z171100001017017018) and the CAMS Innovation Fund for Medical Sciences Project (No. 2016-I2M-3-005). Another source of funding for this work was the National Major Research and Development Programs of the Ministry of Science and Technology of the People's Republic of China during the $13^{\text {th }}$ Five-Year Plan Period (No. 2017YFC1308602).

\section{Footnote}

Reporting Checklist: The authors have completed the PRISMA reporting checklist. Available at http://dx.doi. org/10.21037/atm-20-5606

Conflicts of Interest: All authors have completed the ICMJE uniform disclosure form (available at http://dx.doi. org/10.21037/atm-20-5606). The authors have no conflicts of interest to declare.

Ethical Statement: The authors are accountable for all aspects of the work in ensuring that questions related to the accuracy or integrity of any part of the work are appropriately investigated and resolved.

Open Access Statement: This is an Open Access article distributed in accordance with the Creative Commons Attribution-NonCommercial-NoDerivs 4.0 International License (CC BY-NC-ND 4.0), which permits the noncommercial replication and distribution of the article with the strict proviso that no changes or edits are made and the original work is properly cited (including links to both the formal publication through the relevant DOI and the license). See: https://creativecommons.org/licenses/by-ncnd/4.0/.

\section{References}

1. Christenson ES, Jaffee E, Azad NS. Current and emerging therapies for patients with advanced pancreatic ductal adenocarcinoma: a bright future. Lancet Oncol 2020;21:e135-e145.

2. Siegel RL, Miller KD, Jemal A. Cancer statistics, 2019. CA Cancer J Clin 2019;69:7-34.

3. Gillen S, Schuster T, zum Buschenfelde CM, et al. Preoperative/Neoadjuvant Therapy in Pancreatic Cancer: A Systematic Review and Meta-analysis of Response and Resection Percentages. PLoS Med 2010;7:e1000267.

4. Hartwig W, Hackert T, Hinz U, et al. Pancreatic cancer surgery in the new millennium: better prediction of outcome. Ann Surg 2011;254:311-9.

5. Singh S, Tang SJ, Sreenarasimhaiah J, et al. The clinical utility and limitations of serum carbohydrate antigen (CA19-9) as a diagnostic tool for pancreatic cancer and cholangiocarcinoma. Dig Dis Sci 2011;56:2491-6.

6. Chen Y, Gao SG, Chen JM, et al. Serum CA242, CA199, CA125, CEA, and TSGF are Biomarkers for the Efficacy and Prognosis of Cryoablation in Pancreatic Cancer Patients. Cell Biochem Biophys 2015;71:1287-91.

7. Luo G, Liu C, Guo M, et al. Potential Biomarkers in Lewis Negative Patients With Pancreatic Cancer. Ann Surg 2017;265:800-5.

8. Byrd JC, Bresalier RS. Mucins and mucin binding proteins in colorectal cancer. Cancer Metastasis Rev 2004;23:77-99.

9. Huang J, Che MI, Huang YT, et al. Overexpression of MUC15 activates extracellular signal-regulated kinase 1/2 and promotes the oncogenic potential of human colon cancer cells. Carcinogenesis 2009;30:1452-8.

10. Singh PK, Hollingsworth MA. Cell surface-associated 
mucins in signal transduction. Trends Cell Biol 2006; 16:467-76.

11. Banerjee S, Mujumdar N, Dudeja V, et al. MUC1c regulates cell survival in pancreatic cancer by preventing lysosomal permeabilization. PLoS One 2012;7:e43020.

12. Awaya H, Takeshima Y, Yamasaki M, et al. Expression of MUC1, MUC2, MUC5AC, and MUC6 in atypical adenomatous hyperplasia, bronchioloalveolar carcinoma, adenocarcinoma with mixed subtypes, and mucinous bronchioloalveolar carcinoma of the lung. Am J Clin Pathol 2004;121:644-53.

13. He YF, Zhang MY, Wu X, et al. High MUC2 expression in ovarian cancer is inversely associated with the M1/M2 ratio of tumor-associated macrophages and patient survival time. PLoS One 2013;8:e79769.

14. Patel DS, Khandeparkar SGS, Joshi AR, et al. Immunohistochemical Study of MUC1, MUC2 and MUC5AC Expression in Primary Breast Carcinoma. J Clin Diagn Res 2017;11:EC30-EC34.

15. Zhang H, Liu Y, Xie H, et al. High mucin 5AC expression predicts adverse postoperative recurrence and survival of patients with clear-cell renal cell carcinoma. Oncotarget 2017;8:59777-90.

16. Jonckheere N, Skrypek N, Van Seuningen I. Mucins and pancreatic cancer. Cancers (Basel) 2010;2:1794-812.

17. Pillai K, Akhter J, Chua TC, et al. Anticancer property of bromelain with therapeutic potential in malignant peritoneal mesothelioma. Cancer Invest 2013;31:241-50.

18. Swartz MJ, Batra SK, Varshney GC, et al. MUC4 expression increases progressively in pancreatic intraepithelial neoplasia. Am J Clin Pathol 2002;117:791-6.

19. Park HU, Kim JW, Kim GE, et al. Aberrant expression of MUC3 and MUC4 membrane-associated mucins and sialyl Le(x) antigen in pancreatic intraepithelial neoplasia. Pancreas 2003;26:e48-54.

20. Yonezawa S, Higashi M, Yamada N, et al. Significance of mucin expression in pancreatobiliary neoplasms. J Hepatobiliary Pancreat Sci 2010;17:108-24.

21. Kaur S, Smith LM, Patel A, et al. A Combination of MUC5AC and CA19-9 Improves the Diagnosis of Pancreatic Cancer: A Multicenter Study. Am J Gastroenterol 2017;112:172-83.

22. Takaori K, Hruban RH, Maitra A, et al. Pancreatic intraepithelial neoplasia. Pancreas 2004;28:257-62.

23. Moher D, Liberati A, Tetzlaff J, et al. Preferred reporting items for systematic reviews and meta-analyses: the PRISMA statement. PLoS Med 2009;6:e1000097.
24. Whiting P, Rutjes AW, Reitsma JB, et al. The development of QUADAS: a tool for the quality assessment of studies of diagnostic accuracy included in systematic reviews. BMC Med Res Methodol 2003;3:25.

25. Deville WL, Buntinx F, Bouter LM, et al. Conducting systematic reviews of diagnostic studies: didactic guidelines. BMC Med Res Methodol 2002;2:9.

26. Zamora J, Abraira V, Muriel A, et al. Meta-DiSc: a software for meta-analysis of test accuracy data. BMC Med Res Methodol 2006;6:31.

27. Higgins JP, Thompson SG, Deeks JJ, et al. Measuring inconsistency in meta-analyses. BMJ 2003;327:557-60.

28. Lau J, Ioannidis JP, Schmid CH. Quantitative synthesis in systematic reviews. Ann Intern Med 1997;127:820-6.

29. Terada T, Ohta T, Sasaki M, et al. Expression of MUC apomucins in normal pancreas and pancreatic tumours. $\mathrm{J}$ Pathol 1996;180:160-5.

30. Masaki Y, Oka M, Ogura Y, et al. Sialylated MUC1 mucin expression in normal pancreas, benign pancreatic lesions, and pancreatic ductal adenocarcinoma. Hepatogastroenterology 1999;46:2240-5.

31. Osako M, Yonezawa S, Siddiki B, et al. Immunohistochemical study of mucin carbohydrates and core proteins in human pancreatic tumors. Cancer 1993;71:2191-9.

32. Yonezawa S, Nakamura A, Horinouchi M, et al. The expression of several types of mucin is related to the biological behavior of pancreatic neoplasms. J Hepatobiliary Pancreat Surg 2002;9:328-41.

33. Luttges J, Zamboni G, Longnecker D, et al. The immunohistochemical mucin expression pattern distinguishes different types of intraductal papillary mucinous neoplasms of the pancreas and determines their relationship to mucinous noncystic carcinoma and ductal adenocarcinoma. Am J Surg Pathol 2001;25:942-8.

34. Adsay NV, Merati K, Andea A, et al. The dichotomy in the preinvasive neoplasia to invasive carcinoma sequence in the pancreas: differential expression of MUC1 and MUC2 supports the existence of two separate pathways of carcinogenesis. Mod Pathol 2002;15:1087-95.

35. Chhieng DC, Benson E, Eltoum I, et al. MUC1 and MUC2 expression in pancreatic ductal carcinoma obtained by fine-needle aspiration. Cancer 2003;99:365-71.

36. Kim GE, Bae HI, Park HU, et al. Aberrant expression of MUC5AC and MUC6 gastric mucins and sialyl Tn antigen in intraepithelial neoplasms of the pancreas. Gastroenterology 2002;123:1052-60.

37. Terris B, Dubois S, Buisine MP, et al. Mucin gene 
expression in intraductal papillary-mucinous pancreatic tumours and related lesions. J Pathol 2002;197:632-7.

38. Ohuchida K, Mizumoto K, Yamada D, et al. Quantitative analysis of MUC1 and MUC5AC mRNA in pancreatic juice for preoperative diagnosis of pancreatic cancer. Int J Cancer 2006;118:405-11.

39. Tajiri T, Tate G, Inagaki T, et al. Intraductal tubular neoplasms of the pancreas: histogenesis and differentiation. Pancreas 2005;30:115-21.

40. Ueda M, Miura Y, Kunihiro O, et al. MUC1 overexpression is the most reliable marker of invasive carcinoma in intraductal papillary-mucinous tumor (IPMT). Hepatogastroenterology 2005;52:398-403.

41. Zhang H, Lu H, Kong G. Significance of expression of MUC1 in pancreatic tumor tissues. Modern Oncol 2005;13:756-8.

42. Giorgadze TA, Peterman H, Baloch ZW, et al. Diagnostic utility of mucin profile in fine-needle aspiration specimens of the pancreas: an immunohistochemical study with surgical pathology correlation. Cancer 2006;108:186-97.

43. Okada T, Masuda N, Fukai Y, et al. Immunohistochemical expression of 14-3-3 sigma protein in intraductal papillarymucinous tumor and invasive ductal carcinoma of the pancreas. Anticancer Res 2006;26:3105-10.

44. Wang Y, Gao J, Li Z, et al. Diagnostic value of mucins (MUC1, MUC2 and MUC5AC) expression profile in endoscopic ultrasound-guided fine-needle aspiration specimens of the pancreas. Int J Cancer 2007;121:2716-22.

45. Gao Z, Ding Z, Chen Y, et al. The expression of MUC1 in pancreatic cancer tissues. Shijie Huaren Xiaohua Zazhi 2006;14:923-6.

46. Yamasaki H, Ikeda S, Okajima M, et al. Expression and localization of MUC1, MUC2, MUC5AC and small intestinal mucin antigen in pancreatic tumors. Int $\mathrm{J}$ Oncol 2004;24:107-13.

47. Balmana M, Duran A, Gomes C, et al. Analysis of sialylLewis $\mathrm{x}$ on MUC5AC and MUC1 mucins in pancreatic cancer tissues. Int J Biol Macromol 2018;112:33-45.

48. Gomez-Penaloza C, Serrano-Arevalo ML, VillegasGonzalez LF, et al. Addition of analysis of KRAS mutation or immunohistochemistry with MUC1 and carcinoembryonic antigen improves the diagnostic performance of fine needle aspiration cytology for the diagnosis of pancreatic carcinoma. Cytopathology 2019;30:485-91.

49. Shi C, Merchant N, Newsome G, et al. Differentiation of pancreatic ductal adenocarcinoma from chronic pancreatitis by PAM4 immunohistochemistry. Arch Pathol
Lab Med 2014;138:220-8.

50. Sierzega M, Mlynarski D, Tomaszewska R, et al. Semiquantitative immunohistochemistry for mucin (MUC1, MUC2, MUC3, MUC4, MUC5AC, and MUC6) profiling of pancreatic ductal cell adenocarcinoma improves diagnostic and prognostic performance. Histopathology 2016;69:582-91.

51. Sopha SC, Gopal P, Merchant NB, et al. Diagnostic and therapeutic implications of a novel immunohistochemical panel detecting duodenal mucosal invasion by pancreatic ductal adenocarcinoma. Int J Clin Exp Pathol 2013;6:2476-86.

52. Andrianifahanana M, Moniaux N, Schmied BM, et al. Mucin (MUC) gene expression in human pancreatic adenocarcinoma and chronic pancreatitis: a potential role of MUC4 as a tumor marker of diagnostic significance. Clin Cancer Res 2001;7:4033-40.

53. Bhardwaj A, Marsh WL Jr, Nash JW, et al. Double immunohistochemical staining with MUC4/p53 is useful in the distinction of pancreatic adenocarcinoma from chronic pancreatitis: a tissue microarray-based study. Arch Pathol Lab Med 2007;131:556-62.

54. Urey C, Andersson B, Ansari D, et al. Low MUC4 expression is associated with survival benefit in patients with resectable pancreatic cancer receiving adjuvant gemcitabine. Scand J Gastroenterol 2017;52:595-600.

55. Jhala N, Jhala D, Vickers SM, et al. Biomarkers in Diagnosis of pancreatic carcinoma in fine-needle aspirates. Am J Clin Pathol 2006;126:572-9.

56. Yonezawa S, Horinouchi M, Osako M, et al. Gene expression of gastric type mucin (MUC5AC) in pancreatic tumors: its relationship with the biological behavior of the tumor. Pathol Int 1999;49:45-54.

57. Streppel MM, Vincent A, Mukherjee R, et al. Mucin 16 (cancer antigen 125) expression in human tissues and cell lines and correlation with clinical outcome in adenocarcinomas of the pancreas, esophagus, stomach, and colon. Hum Pathol 2012;43:1755-63.

58. Jiang K, Tan E, Sayegh Z, et al. Cancer Antigen 125 (CA125, MUC16) Protein Expression in the Diagnosis and Progression of Pancreatic Ductal Adenocarcinoma. Appl Immunohistochem Mol Morphol 2017;25:620-3.

59. Haridas D, Chakraborty S, Ponnusamy MP, et al. Pathobiological implications of MUC16 expression in pancreatic cancer. PLoS One 2011;6:e26839.

60. Coppin L, Benomar K, Corfiotti F, et al. CA-125, but not galectin-3, complements CA 19-9 for discriminating ductal adenocarcinoma versus non-malignant pancreatic diseases. 
Pancreatology 2016;16:115-20.

61. Bassi C, Salvia R, Gumbs AA, et al. The value of standard serum tumor markers in differentiating mucinous from serous cystic tumors of the pancreas: CEA, Ca 19-9, Ca 125, Ca 15-3. Langenbecks Arch Surg 2002;387:281-5.

62. Ibrahim NK, Yariz KO, Bondarenko I, et al.

Randomized phase II trial of letrozole plus anti-MUC1 antibody AS1402 in hormone receptor-positive locally advanced or metastatic breast cancer. Clin Cancer Res 2011;17:6822-30.

63. Saeki N, Sakamoto H, Yoshida T. Mucin 1 gene (MUC1) and gastric-cancer susceptibility. Int J Mol Sci 2014;15:7958-73.

64. Kitamoto S, Yokoyama S, Higashi M, et al. MUC1 enhances hypoxia-driven angiogenesis through the regulation of multiple proangiogenic factors. Oncogene 2013;32:4614-21.

65. de Bono JS, Rha SY, Stephenson J, et al. Phase I trial of a murine antibody to MUC1 in patients with metastatic cancer: evidence for the activation of humoral and cellular antitumor immunity. Ann Oncol 2004;15:1825-33.

66. Roy LD, Sahraei M, Subramani DB, et al. MUC1 enhances invasiveness of pancreatic cancer cells by inducing epithelial to mesenchymal transition. Oncogene 2011;30:1449-59.

67. Jones CM, Athanasiou T. Summary receiver operating characteristic curve analysis techniques in the evaluation of diagnostic tests. Ann Thorac Surg 2005;79:16-20.

68. Yonezawa S, Sato E. Expression of mucin antigens in human cancers and its relationship with malignancy potential. Pathol Int 1997;47:813-30.

69. Bafna S, Kaur S, Momi N, et al. Pancreatic cancer cells resistance to gemcitabine: the role of MUC4 mucin. Br J Cancer 2009;101:1155-61.

70. Gautam SK, Kumar S, Dam V, et al. MUCIN-4 (MUC4) is a novel tumor antigen in pancreatic cancer immunotherapy. Semin Immunol 2020;47:101391.

71. Iacobuzio-Donahue CA, Ashfaq R, Maitra A, et al. Highly expressed genes in pancreatic ductal adenocarcinomas: a comprehensive characterization and comparison of the transcription profiles obtained from three major technologies. Cancer Res 2003;63:8614-22.

72. Zhang J, Wang Y, Zhao T, et al. Evaluation of serum MUC5AC in combination with CA19-9 for the diagnosis of pancreatic cancer. World J Surg Oncol 2020;18:31.

73. Yin BW, Lloyd KO. Molecular cloning of the CA125 ovarian cancer antigen: identification as a new mucin, MUC16. J Biol Chem 2001;276:27371-5.
74. Yin BW, Dnistrian A, Lloyd KO. Ovarian cancer antigen CA125 is encoded by the MUC16 mucin gene. Int J Cancer 2002;98:737-40.

75. Wang S, You L, Dai M, et al. Mucins in pancreatic cancer: A well-established but promising family for diagnosis, prognosis and therapy. J Cell Mol Med 2020;24:10279-89.

76. Akagi J, Takai E, Tamori Y, et al. CA19-9 epitope a possible marker for MUC-1/Y protein. Int J Oncol 2001;18:1085-91.

77. Mito K, Saito M, Morita K, et al. Clinicopathological and prognostic significance of MUC13 and AGR2 expression in intraductal papillary mucinous neoplasms of the pancreas. Pancreatology 2018;18:407-12.

78. Hinoda Y, Ikematsu Y, Horinochi M, et al. Increased expression of MUC1 in advanced pancreatic cancer. J Gastroenterol 2003;38:1162-6.

79. Hamanaka Y, Suehiro Y, Fukui M, et al. Circulating antiMUC1 IgG antibodies as a favorable prognostic factor for pancreatic cancer. Int J Cancer 2003;103:97-100.

80. Yokoyama S, Higashi M, Kitamoto S, et al. Aberrant methylation of MUC1 and MUC4 promoters are potential prognostic biomarkers for pancreatic ductal adenocarcinomas. Oncotarget 2016;7:42553-65.

81. Sterlacci W, Fiegl M, Veits L, et al. Diagnostic and prognostic impact of mucin 1-6 expression in nonsmall cell lung cancer. Indian J Pathol Microbiol 2018;61:187-91.

82. Betge J, Schneider NI, Harbaum L, et al. MUC1, MUC2, MUC5AC, and MUC6 in colorectal cancer: expression profiles and clinical significance. Virchows Arch 2016;469:255-65.

83. Kesari MV, Gaopande VL, Joshi AR, et al. Immunohistochemical study of MUC1, MUC2 and MUC5AC in colorectal carcinoma and review of literature. Indian J Gastroenterol 2015;34:63-7.

84. Dai W, Liu J, Liu B, et al. Systematical Analysis of the Cancer Genome Atlas Database Reveals EMCN/MUC15 Combination as a Prognostic Signature for Gastric Cancer. Front Mol Biosci 2020;7:19.

(English Language Editor: A. Kassem; Quality Control Editor: J. Gray)

Cite this article as: Wang S, You L, Dai M, Zhao Y. Quantitative assessment of the diagnostic role of mucin family members in pancreatic cancer: a meta-analysis. Ann Transl Med 2021;9(3):192. doi: 10.21037/atm-20-5606 